Article

\title{
Design Low Crosstalk Ring-Slot Array Structure for Label-Free Multiplexed Sensing
}

\author{
Lijun Huang ${ }^{1,2}$, Huiping Tian ${ }^{1, *}$, Jian Zhou ${ }^{1}$ and Yuefeng $\mathrm{Ji}^{1, *}$
}

1 State Key Laboratory of Information Photonics and Optical Communications, School of Information and Communication Engineering, Beijing University of Posts and Telecommunications, Beijing 100876, China; E-Mails: hljnet@163.com (L.H.); zhoujian19890109@sina.com (J.Z.)

2 Department of Physics and Information Engineering, Huaihua University, Huaihua 418008, China

* Authors to whom correspondence should be addressed; E-Mails: hptian@bupt.edu.cn (H.T.); jyf@bupt.edu.cn (Y.J.); Tel.: +86-010-6228-2153 (Y.J.).

Received: 19 June 2014; in revised form: 14 August 2014 / Accepted: 20 August 2014 /

Published: 25 August 2014

\begin{abstract}
We theoretically demonstrate a low crosstalk ring-slot array structure used for label-free multiplexed sensing. The proposed sensors array is based on an array of three ring-slot and input/output line defect coupling waveguides. Each ring-slot cavity has slightly different cavity spacing and different resonant frequency. Results obtained using two dimensional finite-difference time-domain (2D-FDTD) simulation indicate that the resonant frequencies of each sensor unit in response to the refractive index variations are independent. The refractive index sensitivity is $134 \sim 145.5 \mathrm{~nm} / \mathrm{RIU}$ (refractive index unit) and the $Q$ factors more than $10^{4}$ can be achieved. The calculated detect limit lower than $1.13 \times 10^{-4} \mathrm{RIU}$ is obtained. In addition, an extremely small crosstalk lower than $-25.8 \mathrm{~dB}$ is achieved among the array of three ring-slot cavities. The results demonstrate that this multiplexed sensor array is a promising platform for integrated optical devices and enables highly parallel label-free detection.
\end{abstract}

Keywords: integrated optical devices; optical sensing and sensors; low crosstalk; photonic crystals 


\section{Introduction}

Integrated optical devices based on photonic crystals (PhCs) are a promising platform for high density integration of devices which significantly enhance light-matter interaction [1-3]. In recent years, a wide variety of applications have been proposed and demonstrated, including optical switches [4,5], light emitting diodes [6], lasers [7,8], all-optical logic gates [9,10], filters [11,12], power splitters [13,14] and highly efficient modulators [15-17]. In particular, $\mathrm{PhC}$ microcavities of various architectures have attracted significant interests in molecule sensing due to their compact size, low detect limit (DL) and high sensitivity $(S)$ [18-24], thus far, 1D PhC [20,25,26], 2D PhC [27-29], L3 cavities [30], slot waveguides [24], ring cavities [31-33], have been studied widely for sensing, since the size of PhC sensors are approximately three orders of magnitude less than commercial integrated-optic sensors [34]. For these devices, in a solid medium, and liquid samples in close proximity to the cavities' surface, light can be used to detect the alteration of refractive index caused by the evanescent tails of the guided mode inside the cavities. For use as a label-free sensor, higher refractive index (RI) molecules are bound to the sensor surface, superseding the lower refractive index water, and the resonant wavelength shifts as the refractive index of surrounding cavities changes, that is, the surrounding index can be detected by measuring the wavelength shift via a spectrometer. However, most of these designs concentrate on a single sensor and the target numbers of samples probed at one time are relatively small.

Next generation biosensor platforms must have inherently high degrees of multiplexing capabilities [3]. The more the number of disease markers which can be probed, the more information that can be obtained. Multiplexing capability for pathogen detection is required not only to screen against the large numbers of different pathogens which can be differentiated in a given sample but also to provide specific subtype information. For example, subtype information is extremely important in virology for tracking emerging viruses and designing appropriate vaccines [35]. Therefore, $\mathrm{PhC}$ array sensors recently have been proposed and demonstrated to overcome the insufficiency and realize multiple biosensing. Zlatanovic et al. [36] described time-resolved label-free monitoring of protein binding in a physiological buffer using a photonic crystal microcavity sensor of $50 \mu \mathrm{m}^{2}$ total area with an effective detection area of $0.272 \mu \mathrm{m}^{2}$, however, at a concentration of $0.67 \mathrm{nM}$ of probe biomolecules with $\mathrm{K}_{\mathrm{d}} \approx 6 \times 10^{-7} \mathrm{M}$, the resonant wavelength shift is less than $0.05 \mathrm{~nm}$ with a resonant quality $(Q)$ factor of $\sim 300$. Mandal et al. [25] demonstrated a nanoscale optofluidic sensor array based on a silicon waveguide with a $1 \mathrm{D} \mathrm{PhC}$ microcavity for label-free detection of biomolecular interactions in aqueous environments, in which the device exhibits a bulk refractive index sensitivity of $130 \mathrm{~nm} / \mathrm{RIU}$ and a $Q$ factor of around 3000 . In addition, the single notch extinction ratio is only $4 \sim 10 \mathrm{~dB}$. Yang et al. [37] studied theoretically a nanoscale photonic crystal sensor array on monolithic substrates using a side-coupled resonant cavity array, in which the sensitivity of this device is $115.60 \mathrm{~nm} / \mathrm{RIU}$ and a $Q$ factor is less than 3000 on monolithic substrates. Yang et al. [38] investigated theoretically a nanoscale low crosstalk photonic crystal integrated sensor array. The proposed device consists of array of side-coupled $\mathrm{PhC}$ resonant cavities with $Q$ factors over $2 \times 10^{3}$, in which sensitivities of $\sim 100 \mathrm{~nm} / \mathrm{RIU}$ and crosstalk lower than $-4 \mathrm{~dB}$ are observed. Moreover, the sensing signal of each cavity may interact with each other because of the crosstalk of multi-cavity parallel sensing when the number of sensors integrated is increased.

This paper mainly introduces a label-free multiplexed sensor array with very low crosstalk by designing a ring-slot array structure. The proposed device consists of an array of three ring-slot cavities 
with a high $Q$-factor over $10^{4}$. By adjusting the width of the ring-slot, each cavity has different cavity spacing and different resonant peaks, and the resonant peaks are shifted independently when the refractive index surrounding the cavity is altered. By using FDTD calculation, considering the RI of ambient functionalized area varied from 1.330 to 1.377 , the RI sensitivity $(S)$ is $134 \sim 145.5 \mathrm{~nm} / \mathrm{RIU}$, simultaneously the calculated detect limit (DL) lower than $1.13 \times 10^{-4}$ RIU is obtained. In addition, we further study the crosstalk among three sensors when the RI of a sensor is varied and the other two sensors are not, and the maximum crosstalk among three sensors is less than $-25.8 \mathrm{~dB}$, and the crosstalk is, to the best of our knowledge, the lowest ever reported [38]. The results show that this multiplexed sensing array is a promising platform for integrated optical devices and enables highly parallel detection with very low crosstalk.

\section{PhC Integrated Ring-Slot Sensors Array Design}

The nanoscale integrated $\mathrm{PhC}$ ring-slot sensor array (PhCRSSA) is shown in Figure 1. As seen in this figure, triangular lattice air holes are arranged in silicon $\left(n_{\mathrm{si}}=3.48\right)$. The proposed PhCRSSA consists of three ring-slot cavities (PhCRSS1, PhCRSS2, PhCRSS3), in which six air holes are removed and etched a ring-slot in the center of structure and light is coupled input and output in each ring-slot cavity through a line defect waveguide. We simulate the PhCRSSA structure by using the open source FDTD software Meep which further divides space into a discrete grid and the fields are evolved in time using discrete time steps [39]. The grid and the time steps are made finer and finer, and it becomes a closer and closer approximation for the true continuous equations. During the simulation process, the TE Gaussian-pulse source is used and run for several iterations. The resolution is set to 20 (that is, with a grid spacing of $a / 20$, where $a$ represents the lattice constant). One-spatial unit thick perfectly matched layer (PML) which surrounds the simulated area absorbs the fields leaving the simulated region to implement reflections. The light source is placed at the head of the input line defect waveguide and the monitor is placed at the end of three output line defect waveguides. By dividing the output power detected with the monitor by the input power of the source, we obtain the transmittance spectra. The lattice constant $a$ equals to $378 \mathrm{~nm}$ and the air holes radius $r$ is $0.34 a(128.5 \mathrm{~nm})$. In order to obtain slightly different resonant ring-slot cavity spacing and different resonant peaks, the width of each ring-slot in PhCRSSA is designed to have slightly different cavity spacing. As seen in Figure 1, the specific parameters of PhCRSSA are as follows: $\mathrm{W}_{1}=\sqrt{3} a$; PhCRSS1: $\mathrm{W}_{\mathrm{RS} 1}=0.24 a$; PhCRSS2: $\mathrm{W}_{\mathrm{RS} 2}=0.22 a$; PhCRSS3: $\mathrm{W}_{\mathrm{RS} 1}=0.20 \mathrm{a}$. To enhance the transmission efficiencies in the PhCRSS2 and PhCRSS3, based on our experience of adjusting structure and similar to former solution [40], we further alter the position of black air holes and achieve optimized results. The distances of black air holes moved are as follows: $\mathrm{sx}_{1}=0.17 a, \mathrm{sy}_{1}=0.17 a, \mathrm{sx}_{2}=0.17 a, \mathrm{sy}_{2}=0.17 a$. The air holes and ring-slot of each ring-slot resonant cavity in green shadow area are served as functionalized area, that is, the RI of air holes and ring-slot in this area is changed in order to investigate the sensitivity and crosstalk of the array of three sensors.

The output transmission spectra obtained for the optimal three ring-slot cavities are shown in Figure 2a, and the steady-state electric field profile is shown in Figure $2 b$. As seen in Figure 2a, the transmission efficiencies have response peaks at $1519.44 \mathrm{~nm}, 1545.72 \mathrm{~nm}, 1574.65 \mathrm{~nm}$ in a water environment $(\mathrm{RI}=1.330)$ with respect to the red solid line, blue solid line, green solid line, respectively, and the $Q$ factors of the three peaks are equal to $11,029,10,153,10,669$, respectively, and three peaks surrounded 
by black dashed line are chosen to observe the resonant wavelength shift of integrated three sensors when the RI in the functionalized area is varied.

Figure 1. The schematic of nanoscale integrated $\mathrm{PhC}$ ring-slot sensors array (PhCRSSA). The air holes and ring-slot in green area served as functionalized area.

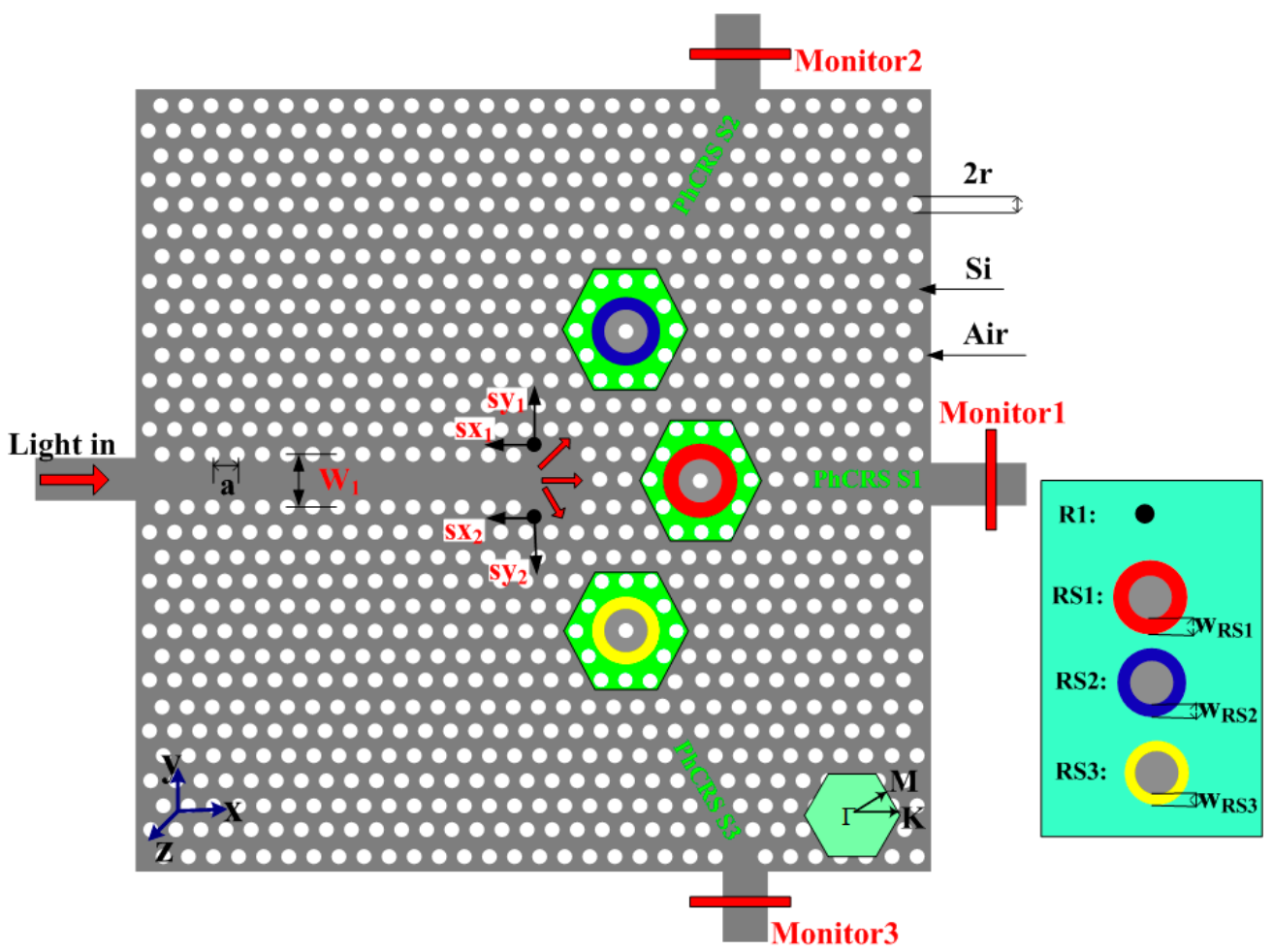

Figure 2. (a) The transmission spectra of three ring-slot cavities (PhCRSS1, PhCRSS2, PhCRSS3) in water environment $(\mathrm{RI}=1.330)$, and observing the resonant peaks of three ring-slot cavities surrounded by black dashed line shift when the RI of functionalized area is changed; (b) The steady-state electric field profile through three ring-slot cavities.

(a)

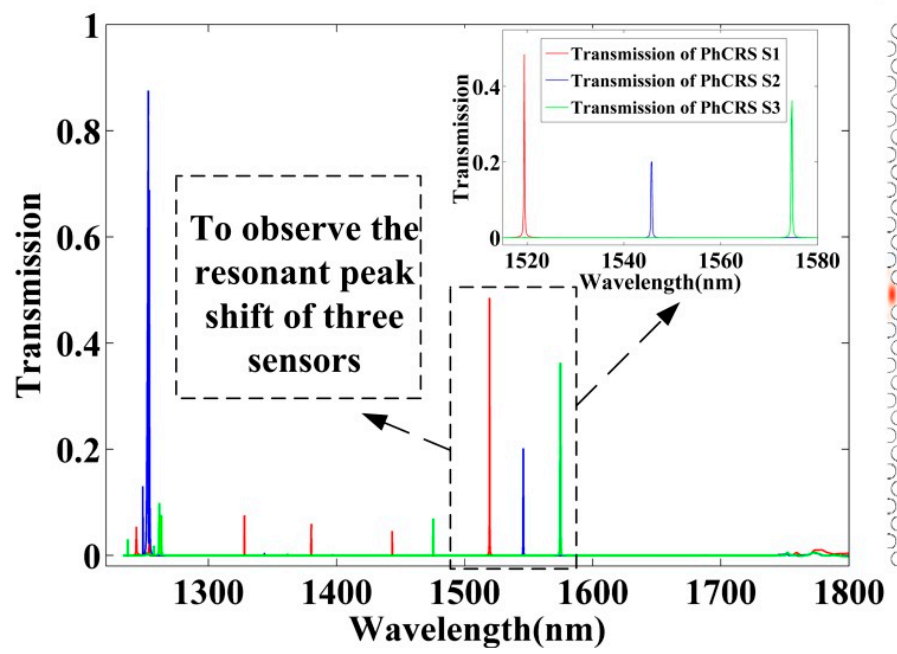

(b)

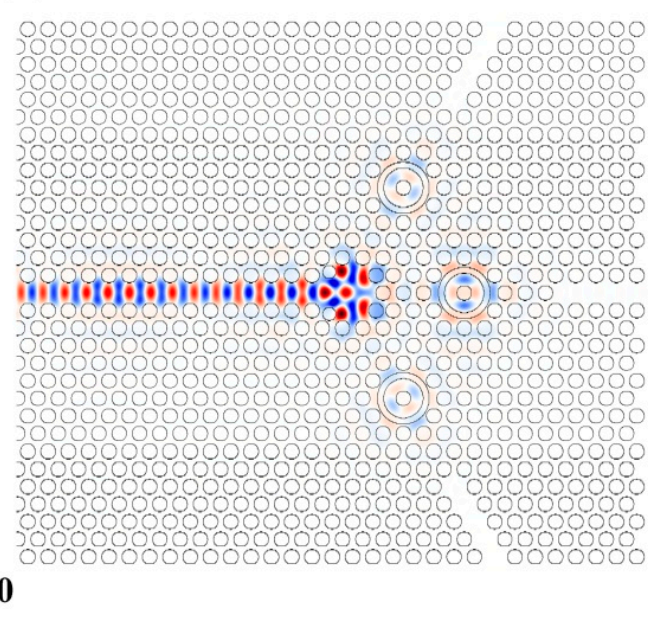




\section{The Properties Analysis of Integrated PhC Ring-Slot Sensors Array}

To analyze the properties of the integrated $\mathrm{PhC}$ ring-slot sensor array in aqueous solution to implement biosensing, the RI of the functionalized area of three sensors is changed, respectively. Figure 3a shows the transmission spectra of three ring-slot cavities (PhCRSS1, PhCRSS2, PhCRSS3) when the RI of functionalized area of one sensor is changed and the others aren't. As shown in Figure 3a, the upper row shows the transmission spectra and the resonant peaks are red-shifted (longer wavelength) when the RI of functionalized area of PhCRSS 1 is changed and the others aren't, and the inset shows the amplified image of the transmission spectra of PhCRSS1 when the RI of functionalized area of PhCRSS1 is changed by $1.330,1.335,1.340,1.345,1.3501,1.355,1.360,1.365,1.370,1.377$, respectively. $Q$ factors of more than $10^{4}$ are achieved for these resonant peaks, and this is higher than ever reported [25,36,37]. We can also find that the transmission spectra of PhCRSS2 and PhCRSS3 are barely transformed when the RI of PhCRSS1 is changed. Similarly, the middle row shows the transmission red-shifted spectra of PhCRSS2 when the RI of PhCRSS2 is transformed, and the lower row shows the red-shifted transmission spectra of PhCRSS3 when the RI of PhCRSS3 is changed.

Figure 3. (a) The transmission spectra of three ring-slot cavities (PhCRSS1, PhCRSS2, $\mathrm{PhCRSS3}$ ) when the RI of functionalized area of one sensor is changed and the others are not;

(b) Wavelength shift $\Delta \lambda$ as a function of the RI of functionalized area increasing.

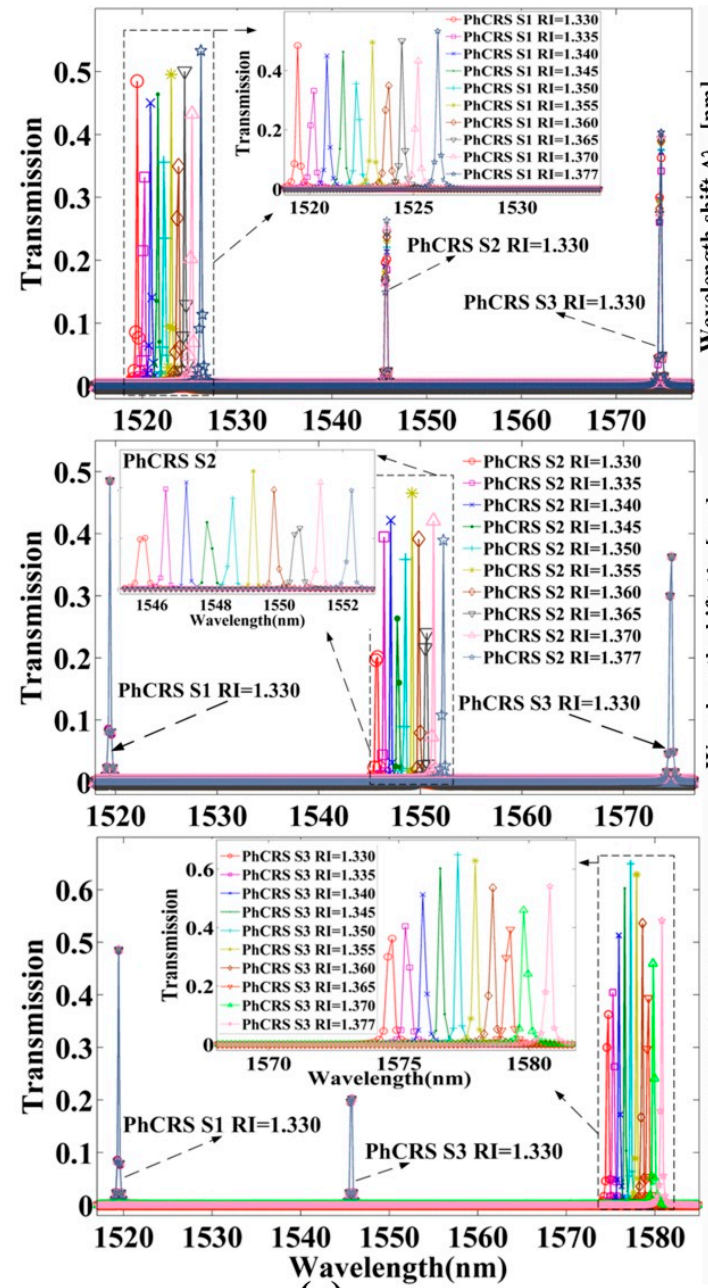

(a)
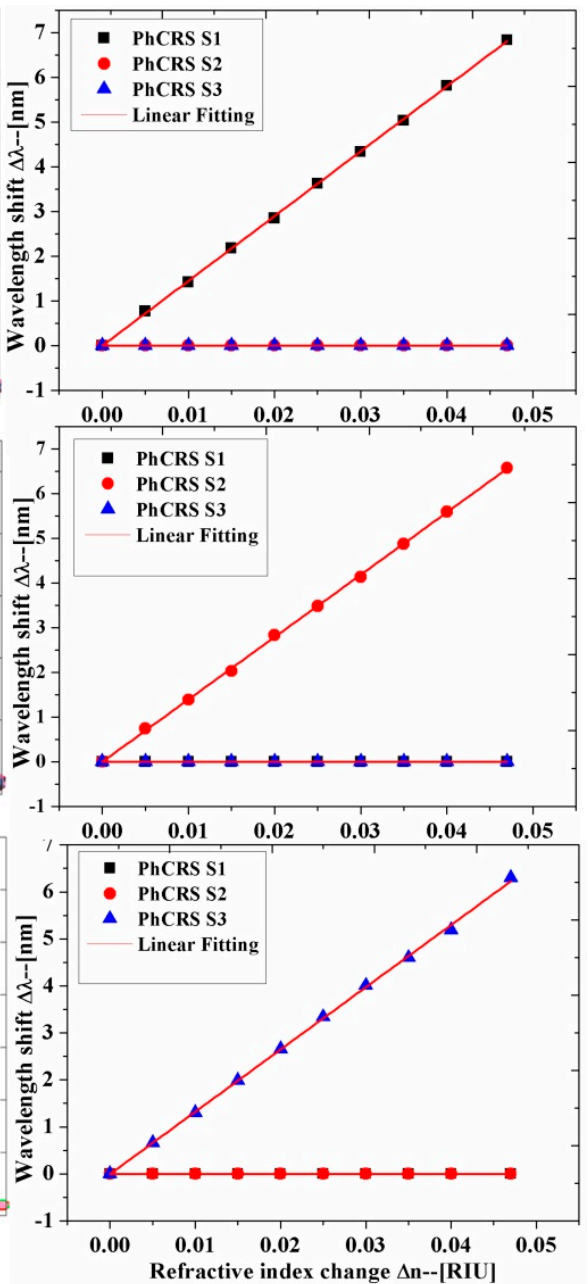

(b) 
The resonant wavelength shift of each ring-slot cavity (PhCRSS1, PhCRSS2, PhCRSS3) as a function of the RI variations of functionalized area is shown in Figure 3b. As seen in this figure, the resonant wavelength shift of each ring-slot cavity is fitted linearly when the RI of functionalized area is varied $(\mathrm{RI}=1.330,1.335,1.340,1.345,1.350,1.355,1.360,1.365,1.370,1.377$, respectively $)$. The sensitivities $S(\mathrm{~S}=\Delta \lambda / \Delta \mathrm{n})$ of the three sensors $S_{1}=145.5 \mathrm{~nm} / \mathrm{RIU}, S_{2}=140 \mathrm{~nm} / \mathrm{RIU}, S_{3}=134 \mathrm{~nm} / \mathrm{RIU}$, are observed, respectively. In order to analyze quantitatively the properties of the sensor array, the sensitivity (S) and the resolution (R) are combined to calculate the detection limit (DL). The DL describes the minimum detectable limit and the sensor resolution $(\mathrm{R})$ characterizes the smallest possible spectral shift that can be accurately measured. In practice, the resolution $(\mathrm{R})$ depends on the experimental noise level, and one-tenth of line-width can easily be resolved in [30]. Here we assume a minimal resolvable wavelength shift to a line-width $\mathrm{R}$ given by $[18,30]$ :

$$
R=\frac{\lambda_{0}}{10 \cdot Q}
$$

where $\lambda_{0}$ represents the resonant wavelength, therefore, the detection limit can be expressed by [18]:

$$
\mathrm{DL}=\frac{R}{S}=\frac{\lambda_{0}}{10 \cdot Q \cdot S}
$$

Based on Equation (2), we can calculate that the detectable minimum change in refractive index is lower than $1.13 \times 10^{-4}$ RIU. References [41] and [42] show the relation that the rate of change with glucose concentration was assumed to be $0.0014 \mathrm{~g} / 100 \mathrm{~mL}$ (or per $1 \%$ concentration) at temperatures of $20{ }^{\circ} \mathrm{C}, 25^{\circ} \mathrm{C}$ and $30{ }^{\circ} \mathrm{C}$. In other words, the RI of a glucose solution is changed from 1.3340 to 1.3480 when the concentration of glucose changes in the range from $0 \%$ to $10 \%$ at $30{ }^{\circ} \mathrm{C}$ temperature, and the variation span lies in the region from 1.330 to 1.350 , This means that the proposed sensor array in theory can be integrated with a fluid flow network to probe glucose molecules.

\section{Crosstalk Analysis of the Integrated PhC Ring-Slot Sensors Array}

The crosstalk of each channel is an important index for an integrated $\mathrm{PhC}$ ring-slot sensor array to implement biosensing. Ideally, what is needed is an integrated sensor that enables highly parallel detection of biomolecule interactions with a high area density of independent sensors that can operate without crosstalk [43]. To evaluate the crosstalk degree of each sensor unit, the maximum crosstalk (MCT) is defined as follows: [38]

$$
M C T=10 \cdot \lg \frac{T}{T_{i}}
$$

where $T_{i}$ represents the minimum transmission efficiency of other sensors at the resonant frequency $\omega_{0}$, and $T$ represents the maximum transmission efficiency of one sensor with the same frequency $\omega_{0}$ when the functionalized area RI of this sensor is varied. The calculated MCT between each sensor is shown in Table 1. 
Table 1. The maximum crosstalk $(\mathrm{dB})$ between the proposed ring-slot sensors array (PhCRSS1, PhCRSS2, PhCRSS3).

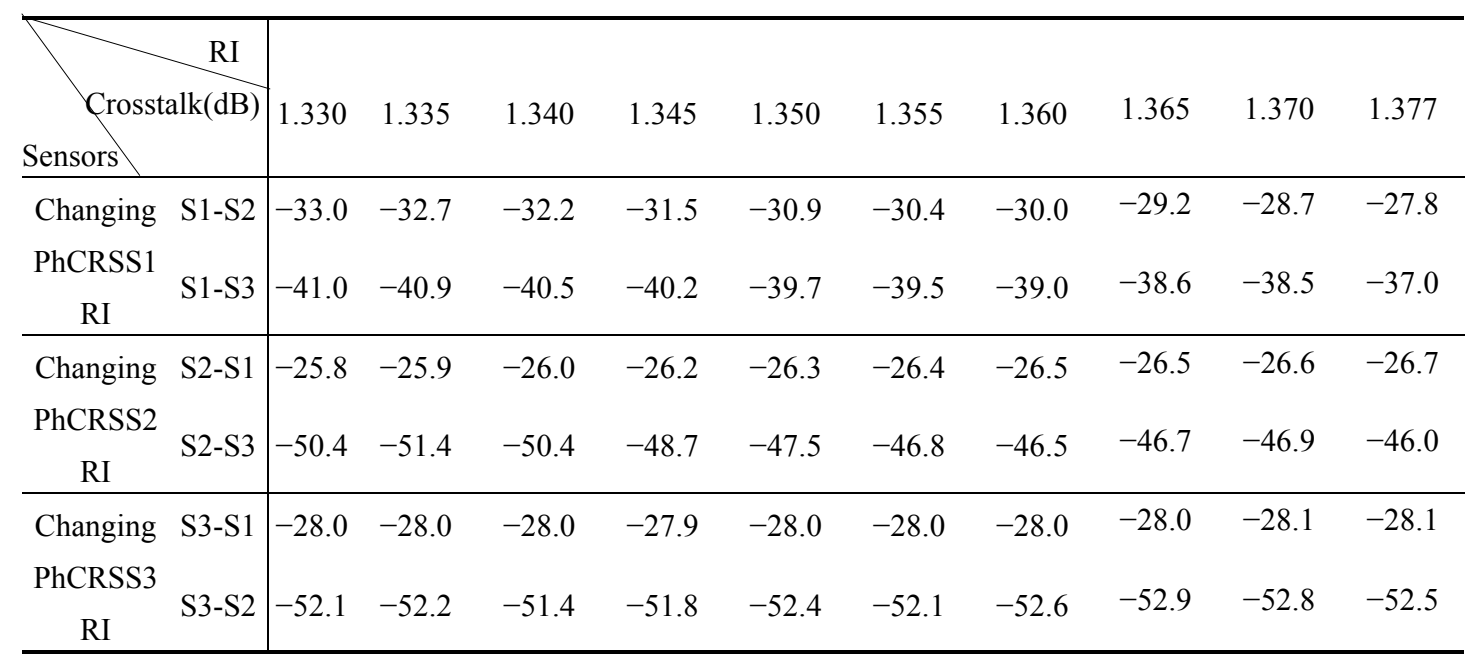

As shown in Table 1, the maximum crosstalk of S1-S2 and S1-S3 are both increased with the increase of functionalized area RI of PhCRSS1, because the resonant peak in Figure 3a is shifted to a longer wavelength and close to the resonant peaks of PhCRSS2 and PhCRSS3 when the functionalized area RI of PhCRSS1 is increased, whereas the crosstalk of S1-S3 is less evident than S1-S2, since the interval of the resonant frequencies of S1-S3 is larger than S1-S2 and the minimum transmission efficiency of PhCRSS3 is higher than PhCRSS2. With the increase of functionalized area RI of PhCRSS2, the crosstalk of S2-S1 slowly become smaller and the crosstalk of S2-S3 become larger, because the resonant peak is red-shifted, resulting in the resonant peak being far away from the resonant peak of PhCRSS1 and approaching the resonant peak of PhCRSS3. The same variation tendency is suitable for the crosstalk of S3-S1 and S3-S2 with the increase of functionalized area RI of PhCRSS3. An evident from the results seen in Table 1, an extremely low crosstalk between each other adjacent sensor units in the integrated $\mathrm{PhC}$ ring-slot sensor array (lower than $-25.8 \mathrm{~dB}$ ) is observed, and this is, to the best of our knowledge, less than ever reported [38]. In theory, for a PhCs-based sensor array structure, the primary source of crosstalk comes from individual sensors as well as nonlinear effects, including stimulated Raman scattering, and Kerr-polarization dependent loss [44-46]. In practice, the free spectrum range, the position of the resonant peak and quality factor are affected due to fabrication imperfections and noise levels in optical sensors [47]. Consequently, the crosstalk among individual sensor should be increased. Generally, the value of crosstalk is more than the theoretical value. Therefore, this low crosstalk feature could be potentially very useful for monolithically integrated and multiplexed sensing.

\section{Conclusions}

We have theoretically designed and demonstrated a label-free integrated ring-slot sensor array structure to implement multiplexed sensing. Different resonant peaks of the array of three ring-slot sensors are obtained by adjusting the width of three ring-slots. Simulation results showed that a $Q$ factor of more than $10^{4}$ and sensitivity $(S) 134 \sim 145.5 \mathrm{~nm} / \mathrm{RIU}$ are achieved when the functionalized area RI is from 1.330 to 1.377 , and simultaneously a detection limit (DL) of less than $1.13 \times 10^{-4} \mathrm{RIU}$ is obtained. In addition, an extremely low crosstalk of less than $-25.8 \mathrm{~dB}$ is achieved among the three sensors. The 
results show that the proposed nanoscale integrated $\mathrm{PhC}$ ring-slot sensors array is a promising platform for monolithically integrated and multiplexed sensing.

\section{Acknowledgments}

This research was supported by NSFC (No. 61372038), National 973 Program (No. 2012CB315705), National 863 Program (No. 2011AA010306) and Fund of State Key Laboratory of Information Photonics and Optical Communications (Beijing University of Posts and Telecommunications), China.

\section{Author Contributions}

Lijun Huang planned the project, supported the FDTD simulations and wrote the manuscript. Huiping Tian planned the project, conceived the study, arranged the architecture and contributed to the writing of the manuscript. Jian Zhou supported the FDTD simulations and suggestion. Yuefeng Ji planed the project, provided suggestion and contributed to the writing of the manuscript. All the authors commented on the manuscript.

\section{Conflicts of Interest}

The authors declare no conflict of interest.

\section{References}

1. Mortensen, N.A.; Xiao, S.S.; Pedersen, J. Liquid-infiltrated photonic crystals: Enhanced light-matter interactions for lab-on-a-chip applications. Microfluid. Nanofluid. 2008, 4, 117-127.

2. Baba, T. Slow light in photonic crystals. Nat. Photo. 2008, 2, 465-473.

3. Erickson, D.; Mandal, S.; Yang, A.H.; Cordovez, B. Nanobiosensors: Optofluidic, electrical and mechanical approaches to biomolecular detection at the nanoscale. Microfluid. Nanofluid. 2008, 4, 33-52.

4. Beggs, D.M.; White, T.P.; O'Faolain, L.; Krauss, T.F. Ultracompact and low-power optical switch based on silicon photonic crystals. Opt. Lett. 2008, 33, 147-149.

5. Vlasov, Y.; Green, W.M.; Xia, F. High-throughput silicon nanophotonic wavelength-insensitive switch for on-chip optical networks. Nat. Photo. 2008, 2, 242-246.

6. Xia, J.; Ikegami, Y.; Shiraki, Y.; Usami, N.; Nakata, Y. Strong resonant luminescence from Ge quantum dots in photonic crystal microcavity at room temperature. Appl. Phys. Lett. 2006, doi:10.1063/1.2386915.

7. Sulkin, J.D.; Choquette, K.D. Hybrid integration of photonic crystal membrane lasers via postprocess bonding. IEEE Photon. J. 2011, 3, 375-380.

8. Zhou, W.; Ma, Z. Breakthroughs in Photonics 2012: Breakthroughs in nanomembranes and nanomembrane lasers. IEEE Photon. J. 2013, doi:10.1109/JPHOT.2013.2250942.

9. O’Brien, J.L.; Pryde, G.J.; White, A.G.; Ralph, T.C.; Branning, D. Demonstration of an all-optical quantum controlled-NOT gate. Nature 2003, 426, 264-267.

10. Xu, Q.; Lipson, M. All-optical logic based on silicon microring resonators. Opt. Express 2007, 15, 924-929. 
11. Wu, Y.; Shih, T.; Lee, J. High-quality-factor filter based on a photonic crystal ring resonator for wavelength division multiplexing applications. Appl. Opt. 2009, 48, F24-F30.

12. Zheng, Y.; Yu, Q.; Tao, K.; Ouyang, Z. All-optical tunable filters based on optomechanical effects in two-dimensional photonic crystal cavities. Opt. Lett. 2013, 38, 4362-4365.

13. Zabelin, V.; Dunbar, L.A.; Thomas, N.L.; Houdre, R.; Kotlyar, M.V.; O’Faolain, L.; Krauss, T.F. Self-collimating photonic crystal polarization beam splitter. Opt. Lett. 2007, 32, 530-532.

14. Yang, D.; Tian, H.; Ji, Y. High-bandwidth and low-loss photonic crystal power-splitter with parallel output based on the integration of Y-junction and waveguide bends. Opt. Commun. 2012, 285, 3752-3757.

15. Wang, X.; Chakravarty, S.; Lee, B.-S.; Lin, C.; Chen, R.T. Ultra-efficient control of light transmission through photonic potential barrier modulation. Opt. Lett. 2009, 34, 3202-3204.

16. Hosseini, A.; Xu, X.; Subbaraman, H.; Lin, C.; Rahimi, S.; Chen, R.T. Large optical spectral range dispersion engineered silicon-based photonic crystal waveguide modulator. Opt. Express 2012, 20, $12318-12325$.

17. Zhang, X.; Hosseini, A.; Lin, X.; Subbaraman, H.; Chen, R.T. Polymer-based Hybrid Integrated Photonic Devices for Silicon On-chip Modulation and Board-Level Optical Interconnects. IEEE J. Sel. Top. Quantum Electron. 2013, doi:10.1109/JSTQE.2013.2268386.

18. White, I.M.; Fan, X. On the performance quantification of resonant refractive index sensors. Opt. Express 2008, 16, 1020-1028.

19. Zhu, J.; Ozdemir, S.K.; Xiao, Y.-F.; Li, L.; He, L.; Chen, D.; Yang, L. On-chip single nanoparticle detection and sizing by mode splitting in an ultrahigh-Q microresonator. Nat. Photon. 2010, 4, $46-49$.

20. Quan, Q.; Vollmer, F.; Burgess, I.B.; Deotare, P.B.; Frank, I.W.; Sindy, T.; Tang, K.Y.; Illic, R.; Loncar, M. Ultrasensitive on-chip photonic crystal nanobeam sensor using optical bistability. In Proceedings of Quantum Electronics and Laser Science Conference (QELS), Baltimore, MD, USA, 1-6 May 2011.

21. Kita, S.; Hachuda, S.; Otsuka, S.; Endo, T.; Imai, Y.; Nishijima, Y.; Misawa, H.; Baba, T. Super-sensitivity in label-free protein sensing using a nanoslot nanolaser. Opt. Express 2011, 19, 17683-17690.

22. Lai, W.; Chakravarty, S.; Zou, Y.; Chen, R.T. Silicon nano-membrane based photonic crystal microcavities for high sensitivity bio-sensing. Opt. Lett. 2012, 37, 1208-1210.

23. Zou, Y.; Chakravarty, S.; Lai, W.; Lin, C.; Chen, R.T. Methods to array photonic crystal microcavities for high throughput high sensitivity biosensing on a silicon-chip based platform. Lab Chip 2012, 12, 2309-2312.

24. Yang, D.; Tian, H.; Ji, Y.; Quan, Q. Design of simultaneous high-Q and high-sensitivity photonic crystal refractive index sensors. J. Opt. Soc. Am. B 2013, 30, 2027-2031.

25. Mandal, S.; Erickson, D. Nanoscale optofluidic sensor arrays. Opt. Express 2008, 16, 1623-1631.

26. Eichenfield, M.; Camacho, R.; Chan, J.; Vahala, K.J.; Painter, O. A picogram- and nanometre-scale photonic crystal optomechanical cavity. Nature 2009, 459, 550-555.

27. Chow, E.; Grot, A.; Mirkarimi, L.; Sigalas, M.; Girolami, G. Ultracompact biochemical sensor built with two-dimensional photonic crystal microcavity. Opt. Lett. 2004, 29, 1093-1095. 
28. Altug, H.; Vucković, J. Polarization control and sensing with two-dimensional coupled photonic crystal microcavity arrays. Opt. Lett. 2005, 30, 982-984.

29. Xu, T.; Zhu, N.; Xu, M.Y.C.; Wosinski, L.; Aitchison, J.S.; Ruda, H.E. A pillar-array based two-dimensional photonic crystal microcavity. Appl. Phys. Lett. 2009, doi:10.1063/1.3152245.

30. Dorfner, D.; Zabel, T.; Hürlimann, T.; Hauke, N.; Frandsen, L.; Rant, U.; Abstreiter, G.; Finley, J. Photonic crystal nanostructures for optical biosensing applications. Biosens. Bioelectron. 2009, 24, 3688-3692.

31. Washburn, A.L.; Gunn, L.C.; Bailey, R.C. Label-free quantitation of a cancer biomarker in complex media using silicon photonics microring resonators. Anal. Chem. 2009, 81, 9499-9506.

32. Mai, T.; Hsiao, F.; Lee, C.; Xiang, W.; Chen, C.; Choi, W. Optimization and comparison of photonic crystal resonators for silicon microcantilever sensors. Sens. Actuators A Phys. 2011, 165, $16-25$.

33. Olyaee, S.; Najafgholinezhad, S. Computational study of a label-free biosensor based on a photonic crystal nanocavity resonator. Appl. Opt. 2013, 52, 7206-7213.

34. Wang, Y.; Wang, H.; Xue, Q.; Zheng, W. Photonic crystal self-collimation sensor. Opt. Express 2012, 20, 12111-12118.

35. Dawson, E.D.; Moore, C.L.; Smagala, J.A.; Dankbar, D.M.; Mehlmann, M.; Townsend, M.B.; Smith, C.B.; Cox, N.J.; Kuchta, R.D.; Rowlen, K.L. MChip: A tool for influenza surveillance. Anal. Chem. 2006, 78, 7610-7615.

36. Zlatanovic, S.; Mirkarimi, L.; Sigalas, M.; Bynum, M.; Chow, E.; Robotti, K.; Burr, G.; Esener, S.; Grot, A. Photonic crystal microcavity sensor for ultracompact monitoring of reaction kinetics and protein concentration. Sens. Actuators B Chem. 2009, 141, 13-19.

37. Yang, D.; Tian, H.; Ji, Y. Nanoscale photonic crystal sensor arrays on monolithic substrates using side-coupled resonant cavity arrays. Opt. Express 2011, 19, 20023-20034.

38. Yang, D.; Tian, H.; Ji, Y. Nanoscale low crossstalk photonic crystal integrated sensor array. IEEE Photon. J. 2014, doi:10.1109/JPHOT.2014.2302805.

39. Oskooi, A.F.; Roundy, D.; Ibanescu, M.; Bermel, P.; Joannopoulos, J.D.; Johnson, S.G. MEEP: A flexible free-software package for electromagnetic simulations by the FDTD method. Comput. Phys. Commun. 2010, 181, 687-702.

40. Zhou, J.; Tian, H.; Yang, D.; Liu, Y.; Liu, Q.; Ji, Y. Ultra-broadband and ultra-low-loss photonic crystal with band-flatness waveguide $60^{\circ}$ bend obtained based on lattice-shifted optimization. Opt. Commun. 2014, 322, 227-233.

41. Weast, R.C.; Astle, M.J.; Beyer, W.H. Handbook of Chemistry and Physics; CRC Press: Boca Raton, FL, USA, 1986-1987; pp. E374-E375.

42. Liu, Y.; Hering, P.; Scully, M.O. An integrated optical sensor for measuring glucose concentration. Appl. Phys. B 1992, 54, 18-23.

43. Choi, C.J.; Cunningham, B.T. Single-step fabrication and characterization of photonic crystal biosensors with polymer microfluidic channels. Lab Chip 2006, 6, 1373-1380.

44. Keiser, G.E. A review of WDM technology and applications. Opt. Fiber Technol. 1999, 5, 3-39.

45. Phillips, M.R.; Ott, D.M. Crosstalk due to optical fiber nonlinearities in WDM CATV lightwave systems. J. Light. Technol. 1999, 17, 1782-1792. 
46. Yang, F.S.; Marhic, M.E.; Kazovsky, L.G. Nonlinear crosstalk and two countermeasures in SCM-WDM optical communication systems. J. Light. Technol. 2000, 18, 512-520.

47. Fussell, D.P.; Hughes, S.; Dignam, M.M. Influence of fabrication disorder on the optical properties of coupled-cavity photonic crystal waveguides. Phys. Rev. B 2008, doi:10.1103/PhysRevB.78.144201.

(C) 2014 by the authors; licensee MDPI, Basel, Switzerland. This article is an open access article distributed under the terms and conditions of the Creative Commons Attribution license (http://creativecommons.org/licenses/by/3.0/). 